\title{
Research Article \\ Effect of Mechanical Stress on Magnetic States and Hysteresis Characteristics of a Two-Phase Nanoparticles System
}

\author{
Leonid Lazarevich Afremov and Ilia Gennadievich Ilyushin \\ Far East State University, 8 Sukhanova Street, Vladivostok 690950, Russia \\ Correspondence should be addressed to Ilia Gennadievich Ilyushin; futted@gmail.com
}

Received 28 March 2013; Revised 25 June 2013; Accepted 9 July 2013

Academic Editor: Miguel A. Correa-Duarte

Copyright ( 2013 L. L. Afremov and I. G. Ilyushin. This is an open access article distributed under the Creative Commons Attribution License, which permits unrestricted use, distribution, and reproduction in any medium, provided the original work is properly cited.

\begin{abstract}
In terms of the two-phase nanoparticles model, the effect of mechanical stress on the magnetic state of both uniaxial and multiaxial heterophase magnetic is investigated. The spectrum of critical fields of reversal of phases' magnetic moments was calculated and phase diagrams were drawn to assess the effect of mechanical stress on the degree of metastability of two-phase nanoparticles' magnetic states. By the example of epitaxial cobalt-coated $\gamma-\mathrm{Fe}_{2} \mathrm{O}_{3}$ particles, a theoretical analysis of the effect of uniaxial mechanical stress on the magnetization of a system of noninteracting heterophase nanoparticles is investigated. It was shown that tension reduced and compression increased coercive force $H_{c}$, while the residual saturation magnetization $I_{\mathrm{rs}}$ was not changed under the influence of mechanical stress.
\end{abstract}

\section{Introduction}

One of the factors that influence the magnetization is the heterophase of nanoparticles determining magnetic properties of nanodispersed materials. This heterophase may be specified by various factors. Thus, according to some authors (see, e.g., [1-5]), the formation of adjacent magnetic phases in natural magnetic materials is associated with oxidation or decomposition processes of a solid solution occurring in magnetic-ordered grains. For example, titanomagnetite being the main carriers of magnetic properties of natural materials can be exposed to decay [3] or oxidation $[4,5]$ processes. As a result of titanomagnetite collapse in nanoparticles, the extraction of both titanium depleted and enriched phases ultimately leads to a change in magnetic properties of a natural material. As an example of multiphase artificial magnetic materials can be nanoparticles with core/shell structure. Currently there are many different types of core/shell nanoparticles, such as metal/metal [6-11], nonmetal/metal [12-14], metal/polymer $[15,16]$, nonmetal/nonmetal, polymer/nonmetal, and polymer/polymer [17]. Development of technology of core/shell nanoparticles allowed for obtaining stable particles of nanometer scale [18-20], the particle size of $1-100 \mathrm{~nm}$ in high demand in many areas, particularly important to its application in medicine [21] and the production of memory storage [22]. Core/shell nanoparticles containing metals (Fe, $\mathrm{Co}$, gold, platinum, palladium, $\mathrm{Mn}, \mathrm{Zn}$, etc.) are used in the manufacture of materials for storage (magnetic memory) and creating the design of microwave and electromagnetic devices [23-25] as well as creating medicines [26], drug delivery [27-29], oncology [30], and biomedicine [31, 32]. Polymer and nonmetallic core/shell nanoparticles are a new class of materials for electronics, for example, the organic lightemitting diodes (OLEDs), organic photovoltaics (OPVs), sensors, and organic field effect transistors (OFETs) [33-35]. Carriers of magnetic memory elements' magnetic properties may be represented by iron particles [36-38] exposed to surface oxidation or magnetic nanoparticles coated with other material, such as cobalt-coated $\gamma$ - $\mathrm{Fe}_{2} \mathrm{O}_{3}$ particles [3941]. According to Mössbauer spectroscopy [36] oxidized coating of iron particles is a mixture of $\gamma-\mathrm{Fe}_{2} \mathrm{O}_{3}$ and $\mathrm{Fe}_{3} \mathrm{O}_{4}$, while the central part is composed of $\alpha$-Fe. The thickness of the coating may be around $10 \%$ of the radius of the particles, and the amount of phase composed of iron oxides is more than 50\%, according to other reports [37]. These particles can be either cubic, spherical $[42,43]$, or very extended [37]. Note that magnetic properties of these nanoparticles can change significantly once they transit to heterophase 
state. Thus, an epitaxial cobalt coating of $\gamma-\mathrm{Fe}_{2} \mathrm{O}_{3}$ particles increases coercive force [41] that is associated by the authors $[44,45]$ with the interfacial exchange interaction. Interfacial exchange interaction can change not only the coercivity but also other magnetic characteristics $[9,46]$. Amorphous magnetic materials obtained in a special way may also refer to multiphase ones. The process of phase formation in such magnets associates with amorphous material transition from metastable to equilibrium state (see, e.g., [47-49]). Nanocrystalline alloys' magnetic matrix that contains grains of other ferromagnets is of special interest here. Exchange interaction between the matrix and grains leads to collective phenomena, which causes a high performance of both magnetically soft and hard materials (e.g., $\mathrm{Fe}, \mathrm{Cu}, \mathrm{Nb}, \mathrm{Si}$, and $\mathrm{B}$ and $\mathrm{Nd}, \mathrm{Fe}$, and $\mathrm{B}$, resp.). Along with the exchange interaction, there is mechanical stress at the interphase interface that is able to alter magnetic states of both phases due to size differences of neighboring phases' lattices. For example, the transformation in iron and its alloys $\gamma \rightarrow \alpha$ is accompanied by a grain extension $(1.5 \%)$ and, as a consequence, by significant deformations. However, the heterogeneity of the interphase interface can lead to a significant reduction of internal stress associated with the $\gamma \rightarrow \alpha$ transformation [50]. It should be noted that the mechanical stresses can have a significant effect on the magnetic properties of nanoparticles [51-55].

Rather detailed theoretical study of magnetic states and magnetization processes of the system of uniaxial two-phase particles is presented in the works [39, 46, 51, 54, 56-59]. In this paper, we attempt to expand the model $[58,59]$ to multiaxis dual-phase particles and studies of the effect of mechanical stress on the magnetic states and magnetic properties of systems of such particles.

\section{The Model of a Uniaxial Two-Phase Nanoparticle}

In contrast to the model of two neighboring plane-parallel phases used in the works [56-59], we use a more plausible model of "inclusion."

(1) Homogeneously magnetized nanoparticle (phase (1)) with the volume $=V$ and the shape of an ellipsoid with the elongation $=q_{1}$ contains a uniformly magnetized ellipsoidal inclusion (phase (2)) with the volume $v=$ $\varepsilon V$, whose long axis coincides with the long axis of the particle oriented along the axis $O Z$ (see Figure 1).

(2) The axes of crystallographic anisotropy $\left\langle k_{A}\right\rangle^{(1)}$ and $\left\langle k_{A}\right\rangle^{(2)}$ of both uniaxial ferromagnets are considered to be parallel to the axis $O Z$, and the vectors of phases' spontaneous magnetization $I_{s}^{(1)}$ and $I_{s}^{(2)}$ are located in the plane $Y O Z$ containing long axes of magnetic phases and make angles $\vartheta^{(1)}$ and $\vartheta^{(2)}$ with and the axis $O Z$, respectively.

(3) The external magnetic field $H$ is placed along the axis $O Z$ as uniaxial mechanical stress $\sigma$.

(4) Thermal fluctuations of phases' magnetic moments may be neglected.

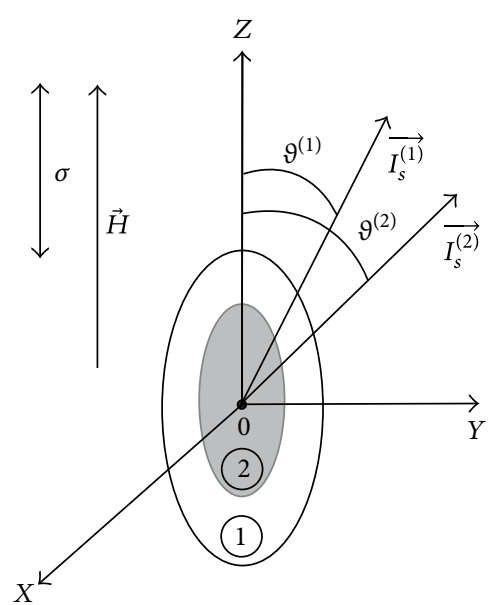

Figure 1: An illustration of the model of a two-phase particle.

We exclude from consideration magnetoelastic interfacial interaction, which may be more or less justified only in case of highly disordered distribution of magnetic atoms in the boundary layer (see, e.g., [50]).

In the accepted approximation, free energy of nanoparticle $F$ located in an external field $H$ can be written as the sum of

(i) crystallographic anisotropy energy:

$$
\begin{aligned}
E_{A}=-\frac{1}{4} & \left\{\left(I_{s}^{(1)}\right)^{2} k_{A}^{(1)}(1-\varepsilon) \cos 2 \vartheta^{(1)}\right. \\
& \left.+\left(I_{s}^{(2)}\right)^{2} k_{A}^{(2)} \varepsilon \cos 2 \vartheta^{(2)}\right\} V,
\end{aligned}
$$

(ii) interaction energy of magnetic moment with its own magnetic field which in accordance with [66] can be represented as

$$
\begin{aligned}
E_{m}=\{ & -\frac{\left(I_{s}^{(1)}\right)^{2}}{4}(1-2 \varepsilon) k_{N}^{(1)} \cos 2 \vartheta^{(1)} \\
& -\frac{\left(I_{s}^{(2)}\right)^{2}}{4} \varepsilon k_{N}^{(2)} \cos 2 \vartheta^{(2)} \\
& +\frac{\varepsilon I_{s}^{(1)} I_{s}^{(2)}}{3}\left(k_{N}^{(2)}-k_{N}^{(1)}\right) \\
& \left.\times\left(\sin \vartheta^{(1)} \sin \vartheta^{(2)}-2 \cos \vartheta^{(1)} \cos \vartheta^{(2)}\right)\right\} V,
\end{aligned}
$$

(iii) energy of magnetoelastic interaction with uniaxial mechanical stress $\sigma$ :

$$
\begin{array}{r}
E_{\sigma}=-\frac{1}{4}\left\{\left(I_{s}^{(1)}\right)^{2} k_{\sigma}^{(1)}(1-\varepsilon) \cos 2 \vartheta^{(1)}\right. \\
\left.+\left(I_{s}^{(2)}\right)^{2} k_{\sigma}^{(2)} \varepsilon \cos 2 \vartheta^{(2)}\right\} V,
\end{array}
$$


(iv) exchange interaction energy across the border, which according to [56] can be defined as

$$
E_{\mathrm{ex}}=-\frac{2 A_{\text {in }}}{\delta} \cos \left(\vartheta^{(1)}-\vartheta^{(2)}\right) s,
$$

(v) interaction energy of magnetic moment with an external magnetic field

$$
E_{H}=-H\left[I_{s}^{(1)}(1-\varepsilon) \cos \vartheta^{(1)}+I_{s}^{(2)} \varepsilon \cos \vartheta^{(2)}\right] V .
$$

In (1)-(5) $k_{A}^{(1,2)}=K_{1} /\left(I_{s}^{(1,2)}\right)^{2}, k_{N}^{(1,2)}$, dimensionless constants of anisotropy and phases' shape anisotropy are $K_{1}$-the first anisotropy constant, and $k_{\sigma}^{(1,2)}=2\left(\lambda_{2}^{(1,2)}+\lambda_{3}^{(1,2)}\right) \sigma /\left(I_{s}^{(1,2)}\right)^{2}$, $\lambda_{2}^{(1,2)}, \lambda_{3}^{(1,2)}$-the second and third magnetostriction constants of a uniaxial crystal; $V$ is the volume of a particle, $s$ is the surface area that separates the phases, $\varepsilon$ is the ratio of the inclusion volume to the volume of the whole particle, $A_{\text {in }}$ is the constant of interfacial exchange interaction, and $\delta$ is the width of the transition region with the order of a constant lattice. Note that the shape anisotropy constant $k_{N}=$ $2 \pi\left(1-3 N_{z}\right)$ is expressed through the demagnetizing factor along the long axis $N_{z}$ depending only on the elongation of the ellipsoid $q$ :

$$
N_{z}=\frac{\left[q \ln \left(q+\sqrt{q^{2}-1}\right)-\sqrt{q^{2}-1}\right]}{\left(q^{2}-1\right)^{3 / 2}} .
$$

Analysis of two-phase particle's magnetic states is a procedure of minimization of the total free energy $(F)$ of a nanoparticle:

$$
\begin{aligned}
F=\{ & -\frac{\left(I_{s}^{(1)}\right)^{2}}{4} K^{(1)} \cos 2 \vartheta^{(1)}-\frac{\left(I_{s}^{(2)}\right)^{2}}{4} K^{(2)} \cos 2 \vartheta^{(2)} \\
& +I_{s}^{(1)} I_{s}^{(2)}\left(-\mathcal{U}_{1} \sin \vartheta^{(1)} \sin \vartheta^{(2)}\right. \\
& \left.+\mathscr{U}_{2} \cos \vartheta^{(1)} \cos \vartheta^{(2)}\right) \\
& \left.-H\left[I_{s}^{(1)}(1-\varepsilon) \cos \vartheta^{(1)}+I_{s}^{(2)} \varepsilon \cos \vartheta^{(2)}\right]\right\} V,
\end{aligned}
$$

where the effective anisotropy constants $K^{(1,2)}$ and the position of are determined by the following formulas:

$$
\begin{gathered}
K^{(1)}=(1-\varepsilon)\left(k_{A}^{(1)}+k_{\sigma}^{(1)}\right)+(1-2 \varepsilon) k_{N}^{(1)}+\varepsilon k_{N}^{(2)}, \\
K^{(2)}=\varepsilon\left(k_{A}^{(2)}+k_{\sigma}^{(2)}+k_{N}^{(2)}\right) .
\end{gathered}
$$

The constants of an interfacial interaction $\mathscr{U}_{1}$ and $\mathscr{U}_{2}$ are expressed through the constants of magnetostatic and exchange interaction of the phases:

$$
\begin{aligned}
& \mathscr{U}_{1}=\varepsilon\left(\frac{\left(k_{N}^{(1)}-k_{N}^{(2)}\right)}{3}+\frac{2 s A_{\mathrm{in}}}{v \delta I_{s}^{(1)} I_{s}^{(2)}}\right), \\
& \mathscr{U}_{2}=\varepsilon\left(\frac{2\left(k_{N}^{(1)}-k_{N}^{(2)}\right)}{3}-\frac{2 s A_{\mathrm{in}}}{v \delta I_{s}^{(1)} I_{s}^{(2)}}\right) .
\end{aligned}
$$

Minimization of free energy (7) by $\vartheta^{(1)}$ and $\vartheta^{(2)}$ leads to a system of equations determining static states of phases' magnetic moments:

$$
\begin{gathered}
\frac{K^{(1)}}{2} \sin 2 \vartheta^{(1)}+j\left[-\mathscr{U}_{1} \cos \vartheta^{(1)} \sin \vartheta^{(2)}\right. \\
\left.-\mathscr{U}_{2} \sin \vartheta^{(1)} \cos \vartheta^{(2)}\right] \\
+\frac{H}{I_{s}^{(1)}}(1-\varepsilon) \sin \vartheta^{(1)}=0, \\
\frac{j K^{(2)}}{2} \sin 2 \vartheta^{(2)}+\left[-\mathscr{U}_{1} \sin \vartheta^{(1)} \cos \vartheta^{(2)}\right. \\
\left.-\mathscr{U}_{2} \cos \vartheta^{(1)} \sin \vartheta^{(2)}\right] \\
+\frac{H}{I_{s}^{(1)}} \varepsilon \sin \vartheta^{(2)}=0,
\end{gathered}
$$

where $j=I_{s}^{(2)} / I_{s}^{(1)}$. The system of (10) allows to determine the main and metastable states of nanoparticles' magnetic moment.

\section{Static States of the Magnetic Moment of Uniaxial Nanoparticle}

The system of equations, within the accuracy of notations, transfers into the system of equations obtained earlier in $[58,59]$, so neglecting thermal fluctuations of the magnetic moment and in the absence of an external magnetic field when $K^{(1)}>0$ and $K^{(2)}>0$ (an "easy axis"), two-phase particles can be in one of the following states:

(i) the first "( $\uparrow \uparrow)$-state": magnetic moments of both phases are parallel and directed along the axis $O Z$;

(ii) the second " $(\uparrow \downarrow)$-state": the phases are antiparallelly magnetized, and the magnetic moment of the first phase $\mathbf{m}^{(1)}$ is directed along the axis $O Z$;

(iii) the third “( $\downarrow \downarrow)$-state": it differs from the first one in the antiparallel orientation of the phases magnetization relative to the axis $O Z$;

(iv) the fourth " $(\downarrow \uparrow)$-state": the magnetic moment of the second phase is directed along the axis $O Z$; the magnetic moment of the first phase is directed against the axis $O Z$.

Besides, if the magnetostatic interaction between the phases dominates the exchange interaction $\mathcal{U}_{2}>0$, the second and fourth states are stable, while the first and third are metastable, since the free energy $F=\left\{-\left|K^{(1)}\right|\left(I_{s}^{(1)}\right)^{2}-\right.$ $\left.\left|K^{(2)}\right|\left(I_{s}^{(2)}\right)^{2}-4 \mathcal{U}_{2} I_{s}^{(1)} I_{s}^{(2)}\right\} V / 4$ of a particle in these states is less than in the first and third, where $F=\left\{-\left|K^{(1)}\right|\left(I_{s}^{(1)}\right)^{2}-\right.$ $\left.\left|K^{(2)}\right|\left(I_{s}^{(2)}\right)^{2}-4 \mathscr{U}_{2} I_{s}^{(1)} I_{s}^{(2)}\right\} V / 4$. Otherwise $\left(\mathscr{U}_{2}<0\right)$, the first and third states are metastable.

The same states are realized in the case when the constants $K^{(1)}$ and $K^{(2)}$ have different signs. If $K^{(1)}<0$ and $K^{(2)}<0$ 
(an "easy plane"), a two-phase particle can be in one of the four states listed above with the phases' magnetic moments perpendicular to the axis $O Z$.

Following (10), if at least one of the constants $K^{(1)}$ or $K^{(2)}$ is positive, then when the external magnetic field obtains a critical value $H_{c}$, the transition from one state to another will occur. For example, the critical field of the transition from the third state to the second or from the first state to the fourth is

$$
H_{c}^{(3 \rightarrow 2)}=H_{c}^{(1 \rightarrow 4)}=\frac{\left|K^{(1)}\right| I_{s}^{(1)}-\mathcal{U}_{2} I_{s}^{(2)}}{1-\varepsilon} .
$$

In much the same way we define the critical field of the transition from the third to the fourth (from the first to the second) state:

$$
H_{c}^{(3 \rightarrow 4)}=H_{c}^{(1 \rightarrow 2)}=\frac{\left|K^{(2)}\right| I_{s}^{(2)}-\mathscr{U}_{2} I_{s}^{(1)}}{\varepsilon}
$$

from the forth to the first (from the second to the third):

$$
H_{c}^{(4 \rightarrow 1)}=H_{c}^{(2 \rightarrow 3)}=\frac{\left|K^{(1)}\right| I_{s}^{(1)}+\mathscr{U}_{2} I_{s}^{(2)}}{1-\varepsilon},
$$

from the second to the first (from the forth to the third):

$$
H_{c}^{(2 \rightarrow 1)}=H_{c}^{(4 \rightarrow 3)}=\frac{\left|K^{(2)}\right| I_{s}^{(2)}+\mathscr{U}_{2} I_{s}^{(1)}}{\varepsilon}
$$

from the third to the first and vice versa:

$$
\begin{aligned}
H_{c}^{(3 \rightarrow 1)} & =H_{c}^{(1 \rightarrow 3)} \\
& =\frac{\left|K^{(1)}\right|\left(I_{s}^{(1)}\right)^{2}+\left|K^{(2)}\right|\left(I_{s}^{(2)}\right)^{2}+2\left(k_{N}^{(2)}-k_{N}^{(1)}\right) I_{s}^{(1)} I_{s}^{(2)}}{(1-\varepsilon) I_{s}^{(1)}+\varepsilon I_{s}^{(2)}}
\end{aligned}
$$

and from the second to the fourth and vice versa:

$$
\begin{aligned}
H_{c}^{(2 \rightarrow 4)} & =H_{c}^{(4 \rightarrow 2)} \\
& =\frac{\left|K^{(1)}\right|\left(I_{s}^{(1)}\right)^{2}+\left|K^{(2)}\right|\left(I_{s}^{(2)}\right)^{2}-2\left(k_{N}^{(2)}-k_{N}^{(1)}\right) I_{s}^{(1)} I_{s}^{(2)}}{\left|(1-\varepsilon) I_{s}^{(1)}-\varepsilon I_{s}^{(2)}\right|} .
\end{aligned}
$$

In the same way we can calculate the spectrum of critical fields when $K^{(1)}<0$ and $K^{(2)}<0$. In this case we can use the relations (11)-(16), having replaced $\mathscr{U}_{2}$ by $-\mathscr{U}_{1}$.

\section{Magnetic States of Multiaxis Two-Phase Nanoparticle}

Let nanoparticle's magnetic phases be represented by the crystals of cubic symmetry with the constants of crystalline anisotropy of the first $K_{1}^{(1,2)}$ and the second $K_{2}^{(1,2)}$ order, respectively. Let us perform a solution of the problem of the magnetic states of a multiphase particle in the framework of the following assumptions.

(1) Crystallographic directions [100], [010], and [001] of the phases coincide with the axes $O X, O Y$, and $O Z$, respectively (see Figure 1), if the first constants $K_{1}^{(1,2)}$ of the phases' crystalline anisotropy are positive. Otherwise $\left(K_{1}^{(1,2)}<0\right)$, we combine the "easy axis" (direction [111]) with the axis OZ.

(2) We use the condition of magnetic uniaxiality of a multiaxis crystal's grain $[57,67]$, the essence of which is that at a certain elongation its shape anisotropy prevails over the crystalline anisotropy. The process of magnetization of these particles is similar to magnetization of uniaxial particles with an effective constant determined by the total free energy. So for materials with $I_{s}>350 \div 500$ (e.g., titanomagnetite or magnetite), the condition of magnetic uniaxiality is satisfied when the elongation $q \geq 1.2$.

As well as in Section 1, we build an expression for the total free energy, which includes

(i) crystalline anisotropy energy, which, according to [62], depending on the sign of the constant of anisotropy has the following form:

(ii)

$$
E_{A}=\left\{\begin{array}{c}
\frac{1}{4} k_{A 1}^{(1)}\left(I_{s}^{(1)}\right)^{2}(1-\varepsilon) \sin ^{2} 2 \vartheta^{(1)}+\frac{1}{4} k_{A 1}^{(2)}\left(I_{s}^{(2)}\right)^{2} \\
\times \varepsilon \sin ^{2} 2 \vartheta^{(2)}, \quad k_{A 1}^{(1)}>0, \quad k_{A 1}^{(2)}>0, \\
\frac{1}{54} k_{A 2}^{(1)}\left(I_{s}^{(1)}\right)^{2}(1-\varepsilon) \sin ^{2} \vartheta^{(1)}\left(1+2 \cos 2 \vartheta^{(1)}\right)^{2} \\
+\frac{1}{54} k_{A 2}^{(2)}\left(I_{s}^{(2)}\right)^{2} \varepsilon \sin ^{2} \vartheta^{(2)}\left(1+2 \cos 2 \vartheta^{(2)}\right)^{2}, \\
\frac{1}{4} k_{A 1}^{(1)}\left(I_{s}^{(1)}\right)^{2}(1-\varepsilon) \sin ^{2} 2 \vartheta^{(1)}<0, \quad k_{A 1}^{(2)}<0, \\
+\frac{1}{54} k_{A 2}^{(2)}\left(I_{s}^{(2)}\right)^{2} \varepsilon \sin ^{2} \vartheta^{(2)}\left(1+2 \cos 2 \vartheta^{(2)}\right)^{2}, \\
k_{A 1}^{(1)}>0, \quad k_{A 1}^{(2)}<0, \\
\frac{1}{54} k_{A 2}^{(1)}\left(I_{s}^{(1)}\right)^{2}(1-\varepsilon) \sin ^{2} \vartheta^{(1)}\left(1+2 \cos 2 \vartheta^{(1)}\right)^{2} \\
+\frac{1}{4} k_{A 1}^{(2)}\left(I_{s}^{(2)}\right)^{2} \varepsilon \sin ^{2} 2 \vartheta^{(2)}, \\
k_{A 1}^{(1)}<0, \quad k_{A 1}^{(2)}>0,
\end{array}\right.
$$

(iii) energy of a grain in the field of mechanical stress

$$
\begin{aligned}
E_{\sigma}= & \frac{1}{2}\left(I_{s}^{(1)}\right)^{2}(1-\varepsilon) \Lambda^{(1)} \sigma \sin ^{2} \vartheta^{(1)} \\
& +\frac{1}{2}\left(I_{s}^{(2)}\right)^{2} \varepsilon \Lambda^{(2)} \sigma \sin ^{2} \vartheta^{(2)}
\end{aligned}
$$

energy of magnetostatic interaction $E_{m}$, interfacial exchange interaction $E_{\mathrm{ex}}$, and energy of the magnetic moment of 
TABLE 1: Magnetic characteristics of two-phase nanoparticles.

\begin{tabular}{lcccc}
\hline & $\mathrm{Fe}_{3} \mathrm{O}_{4}$ & $\mathrm{Fe}_{2.44} \mathrm{Ti}_{0.56} \mathrm{O}_{4}$ & $\mathrm{CoFe}_{2} \mathrm{O}_{4}$ & $\gamma-\mathrm{Fe}_{2} \mathrm{O}_{3}$ \\
\hline$I_{s}, \mathrm{G}$ & $480[60]$ & $151[60]$ & $425[61]$ & $350[44]$ \\
$K_{1}, 10^{5} \mathrm{erg} / \mathrm{cm}^{3}$ & $-1.06[60,61]$ & $-0.7[60]$ & $27[61]$ & $30[61]$ \\
$K_{2}, 10^{5} \mathrm{erg} / \mathrm{cm}^{3}$ & $2.8[60,61]$ & $-0.15[60]$ & $225[63]$ & $52[56]$ \\
$\lambda_{100} \cdot 10^{6}$ & $-20[60,62]$ & $170[60,62]$ & $-335[63,65]$ & $-5^{*}[64]$ \\
$\lambda_{111} \cdot 10^{6}$ & $78[60,62]$ & $92[60,62]$ & & $-5^{*}[64]$ \\
\hline${ }^{*}$ indicates isotopic constants of magnetostriction & &
\end{tabular}

${ }^{*}$ indicates isotopic constants of magnetostriction $\lambda_{s}$.

a particle in the external magnetic field $E_{H}$ defined by the relations (2), (4), and (5), respectively. In the relations (17) and (18) $k_{A 1}^{(1,2)}=K_{1}^{(1,2)} /\left(I_{s}^{(1,2)}\right)^{2}$ and $k_{A 2}^{(1,2)}=K_{2}^{(1,2)} /\left(I_{s}^{(1,2)}\right)^{2}$ are dimensionless constant of crystalline anisotropy of the first or second order of the first or second phase, respectively, $K_{1}^{(1,2)}$ and $K_{2}^{(1,2)}$ are the anisotropy constants of the first and second order of a cubic crystal, and $\Lambda^{(1,2)}=3 \lambda_{100}^{(1,2)} /\left(I_{s}^{(1,2)}\right)^{2}$, where $k_{A 1}^{(1,2)}>0$ and $\Lambda^{(1,2)}=3 \lambda_{111}^{(1,2)} /\left(I_{s}^{(1,2)}\right)^{2}$, where $k_{A 1}^{(1,2)}<0, \lambda_{100}^{(1,2)}$ and $\lambda_{111}^{(1,2)}$ are the constants of phases' magnetostriction.

According to the second condition stated above, magnetic states of a multiaxis two-phase particle are equivalent to states of a uniaxial two-phase particle (see Section 2), whose effective anisotropy constants $K^{(1)}$ and $K^{(2)}$ are expressed through the constants of a multiaxial crystal:

$$
\begin{gathered}
\widetilde{K}^{(1)}=\left\{\begin{array}{cc}
k_{A 1}^{(1)}(1-\varepsilon)+(1-2 \varepsilon) & k_{N}^{(1)}+\varepsilon k_{N}^{(2)} \\
+\Lambda^{(1)} \sigma(1-\varepsilon), & k_{A 1}^{(1)}>0, \\
\frac{k_{A 2}^{(1)}}{3}(1-\varepsilon)+(1-2 \varepsilon) & k_{N}^{(1)}+\varepsilon k_{N}^{(2)} \\
+\Lambda^{(1)} \sigma(1-\varepsilon), & k_{A 1}^{(1)}<0,
\end{array}\right. \\
\widetilde{K}^{(2)}=\left\{\begin{array}{cc}
\varepsilon\left(k_{A 1}^{(2)}+k_{N}^{(2)}+\Lambda^{(2)} \sigma\right), & k_{A 1}^{(2)}>0, \\
\varepsilon\left(\frac{\left.k_{A 2}^{(2)}+k_{N}^{(2)}+\Lambda^{(2)} \sigma\right),}{3}\right. & k_{A 1}^{(2)}<0 .
\end{array}\right.
\end{gathered}
$$

Expressions (11)-(16) and (19) allow us to study the effect of mechanical stress and geometric $\left(q\right.$ and $\varepsilon$ ) and $\left(I_{s}^{(1,2)}\right.$, $\left.k_{A 1,2}^{(1,2)}, \Lambda^{(1,2)}, A_{\text {in }}\right)$ characteristics on the magnetic state of both single-axis and multiaxis crystallographic two-phase nanoparticles.

\section{Diagrams of the Magnetic States of Heterophase Nanoparticles}

For comparison, let us consider two types of two-phase nanoparticles, whose magnetic characteristics are presented in Table 1: relatively low coercitive nanoparticles of magnetite (first phase) that include titanomagnetite $[3,60]\left(\mathrm{Fe}_{3} \mathrm{O}_{4}\right.$ $\mathrm{Fe}_{2.44} \mathrm{Ti}_{0.56} \mathrm{O}_{4}$ ) and high coercitive nanoparticles $\gamma$ - $\mathrm{Fe}_{2} \mathrm{O}_{3}$ (second phase) epitaxially cobalt ferrite coated [39-41] (Co $\mathrm{Fe}_{2} \mathrm{O}_{4}-\gamma-\mathrm{Fe}_{2} \mathrm{O}_{3}$ ).

Critical fields of magnetic reversal (11)-(16) depend essentially on the elongation $q$ and the relative volume of one of the $\varepsilon$ particle's phases. Moreover, for some $q$ and $\varepsilon$ values the critical fields can be negative. Negativity $H_{c}$ should be comprehended as the inability to implement the metastable state controlled by this critical field. This statement may be interpreted in the following way. If an ensemble is made up of particles with different relative sizes $\varepsilon$ and elongation $q$ of the second phase, the point A on the phase diagram $\{\varepsilon, q\}$ (see Figure 2) can be associated with a particle that at $H_{c}>0$ (dark area) can be in either metastable or ground state and only in the ground state if $H_{c}<0$ (light area).

Note that natural limitation of the volume of introduction (of the second phase) $-v \leq V$ and the relation between large semimajor axes of particles' ellipsoids and implementation $a_{2} \leq a_{1}$ impose restrictions on the choice of $q$ and $\varepsilon$. Indeed, if we use expressions for the volume and the long axis of the elongated ellipsoid $\left(v=(4 \pi / 3) q b_{2}^{3}, V=(4 \pi / 3) q_{1} b_{1}^{3}, a_{1}=\right.$ $q_{1} b_{1} a_{2}=q b_{2}, b$-minor axis of the ellipsoid), we can obtain the following relation $q_{1} \varepsilon \leq q \leq q_{1} / \sqrt{\varepsilon}$. Obviously, the range of permissible values of $q$ and $\varepsilon$ depends significantly on the elongation of nanoparticles $q_{1}$ (see Figure 2 ). The maximum value of $q=q_{m}$ and is limited with the value $q_{m}=2 \pi$, since the demagnetizing factor $N_{z}(q)$ decreases rapidly with $q\left(N_{z}(2 \pi) \approx 0.02\right)$ increasing.

In the study of the effect of mechanical stress on the magnetic states and magnetization processes of an ensemble of two-phase nanoparticles we use a dimensionless constant

$$
k_{\sigma}= \begin{cases}\frac{3 \lambda_{100}^{(1)} \sigma}{k_{A 1}^{(1)}\left(I_{s}^{(1)}\right)^{2}}, & k_{A 1}^{(1)}>0 \\ \frac{3 \lambda_{111}^{(1)} \sigma}{\left|k_{A 1}^{(1)}\right|\left(I_{s}^{(1)}\right)^{2}}, & k_{A 1}^{(1)}<0\end{cases}
$$

As shown in Table 1, stress signs $\sigma$ and $k_{\sigma}$ match each other for the particles $\left(\mathrm{Fe}_{3} \mathrm{O}_{4}-\mathrm{Fe}_{2.44} \mathrm{Ti}_{0.56} \mathrm{O}_{4}\right)$, while negative values $(\sigma>0)$ correspond to tensile stress and positive values correspond to compression stress for cobalt-coated particles $\gamma-\mathrm{Fe}_{2} \mathrm{O}_{3}$.

Tables 2 and 3 show the basic diagram and the twophase particles of the metastable states $\left(\mathrm{CoFe}_{2} \mathrm{O}_{4}-\gamma-\mathrm{Fe}_{2} \mathrm{O}_{3}\right)$ and $\left(\mathrm{Fe}_{3} \mathrm{O}_{4}-\mathrm{Fe}_{2.44} \mathrm{Ti}_{0.56} \mathrm{O}_{4}\right)$, respectively, differing magnitude interfacial exchange interaction $A_{\text {in }}$ compressive $(\sigma<0)$ or tensile $(\sigma>0)$ stresses. The particles, which were representative points in the phase diagram $\{\varepsilon, q\}$ in the region highlighted in the dark color, can be both in the ground and metastable states (with a parallel or antiparallel orientation of the magnetic moments of the phases). The points in the light 

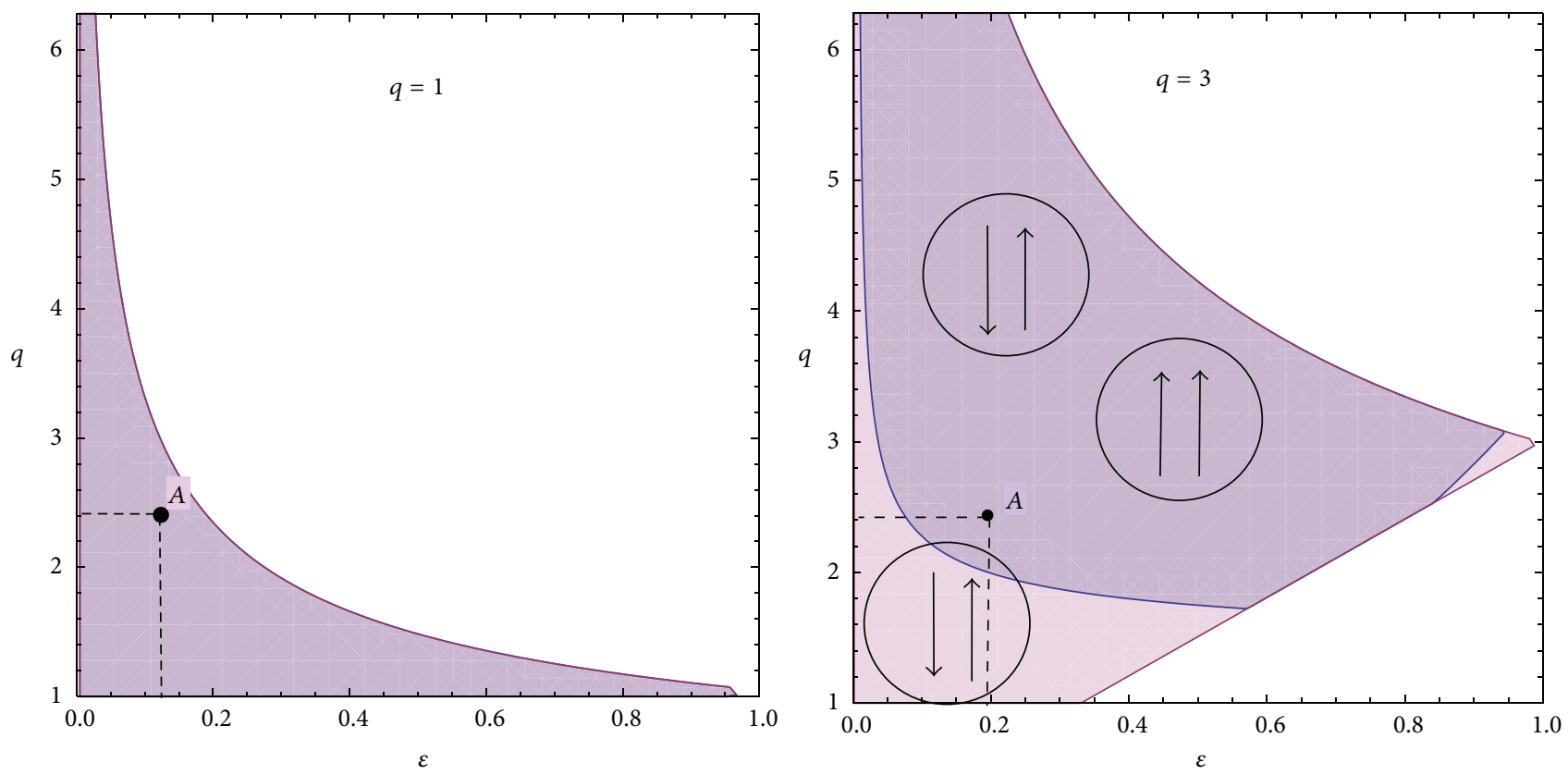

Figure 2: An illustration of the phase diagram $\{\varepsilon, q\}$. A two-phase ellipsoidal nanoparticle with the volume $V$ and elongation $q_{1}$ containing ellipsoidal inclusion with the volume $v=\varepsilon V$ and elongation $q$ corresponds to point $A$. Particles that represent the points in the dark area may be in either ground or metastable state, as their critical fields $H_{c}^{(1 \rightarrow 2)}>0$ in the white area are in the ground state $\left(H_{c}^{(1 \rightarrow 2)}<0\right)$.

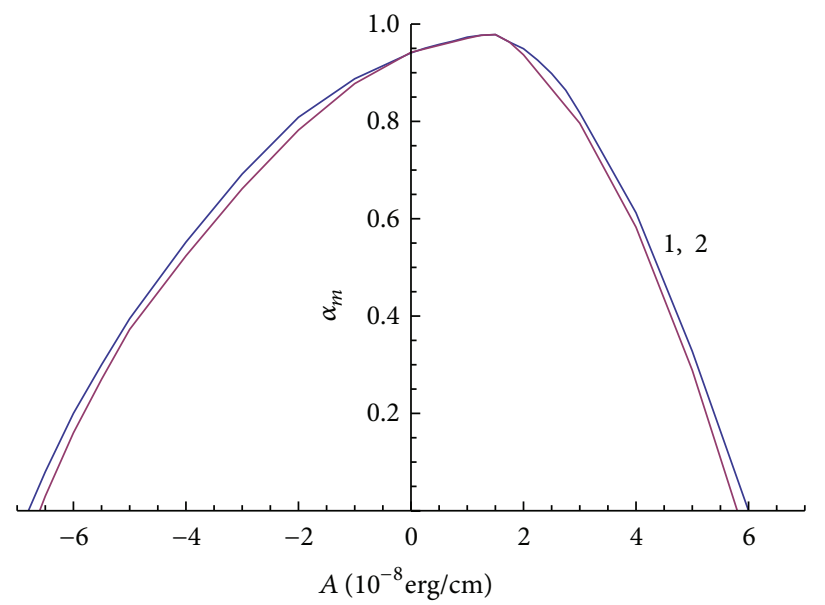

(a)

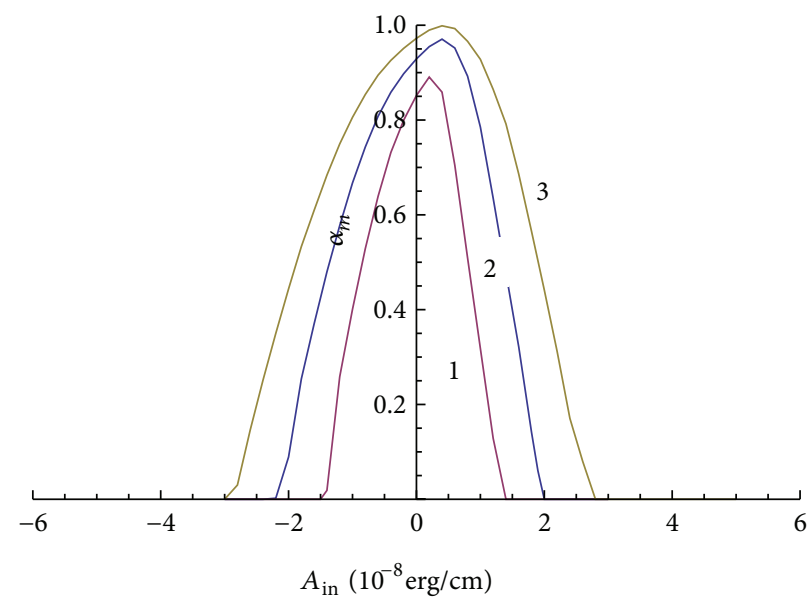

(b)

Figure 3: Dependence of the degree of metastability $\alpha_{m}$ of the system of nanoparticles $\mathrm{CoFe}_{2} \mathrm{O}_{4}-\gamma-\mathrm{Fe}_{2} \mathrm{O}_{3}(\mathrm{a})$ and $\mathrm{Fe}_{3} \mathrm{O}_{4}$ - $\mathrm{Fe}_{2.44} \mathrm{Ti}_{0.56} \mathrm{O}_{4}$ (b) on the value of the interfacial exchange interaction $A_{\text {in }}$ and relative stress $k_{\sigma}$. Curves 1,2 , and 3 are calculated at $k_{\sigma}=-0.8, k_{\sigma}=0$, and $k_{\sigma}=0.8$, respectively.

area correspond to particles in its ground part in Table 1 as footnote. Please check state in which depending on the size of the interfacial exchange interaction $A_{\text {in }}$ magnetic moments are parallel or antiparallel phase with each other. Figures in the second column 2 and the second row of Table 3 illustrate the effect on the metastability $A_{\text {in }}$ biphasic nanoparticles $\sigma=$ 0 .

Let us define the degree of metastability $\alpha_{m}$ of the nanoparticles system as the ratio of the areas: the dark side of the chart to the general-dark and light. Figure 3 shows that the metastable state is realized only at $A_{\text {in min }} \leq A_{\text {in }} \leq A_{\text {in max }}$, and the degree of metastability $\alpha_{m}$ reaches its maximum at a point $A_{\mathrm{in}}^{(m)}$. And, if the magnetic moments of the phases are antiparallel in the ground state at $A_{\text {in }} \leq A_{\text {in }}^{(m)}$, then at $A_{\text {in }}>A_{\text {in }}^{(m)}$ they are parallel.

Table 2 and Figure 3(a) show that the stress does not affect the metastability of cobalt-coated particles $\gamma-\mathrm{Fe}_{2} \mathrm{O}_{3}$. Such a weak dependence of the magnetic states on the stress is associated with high values of anisotropy and shape anisotropy. The effect of mechanical stress on the degree 
TABLE 2: A diagram of the ground (light fill area) and metastable (dark fill area) states of the two-phase elongated $\left(q_{1}=3\right)$ nanoparticles $\left(\mathrm{CoFe}_{2} \mathrm{O}_{4}-\gamma-\mathrm{Fe}_{2} \mathrm{O}_{3}\right)$ system located in the field of mechanical stress $\sigma$. Positive value $k_{\sigma}=0.8$, that is, the right column of the table, corresponds to compressive stress; $k_{\sigma}=-0.8$ column at $k_{\sigma}=0$ no stress (center column) corresponds to tensile stress. Table rows correspond to different values of interfacial interaction $A_{\text {in }}=\alpha \cdot 10^{-8} \mathrm{Erg} / \mathrm{cm}$. Arrows indicate the state of parallel ( $\uparrow$ ) (the first and the third) and antiparallel ( $\left.\uparrow \downarrow\right)$ (the second and the fourth) orientation of the magnetic moments of nanoparticles' phases.

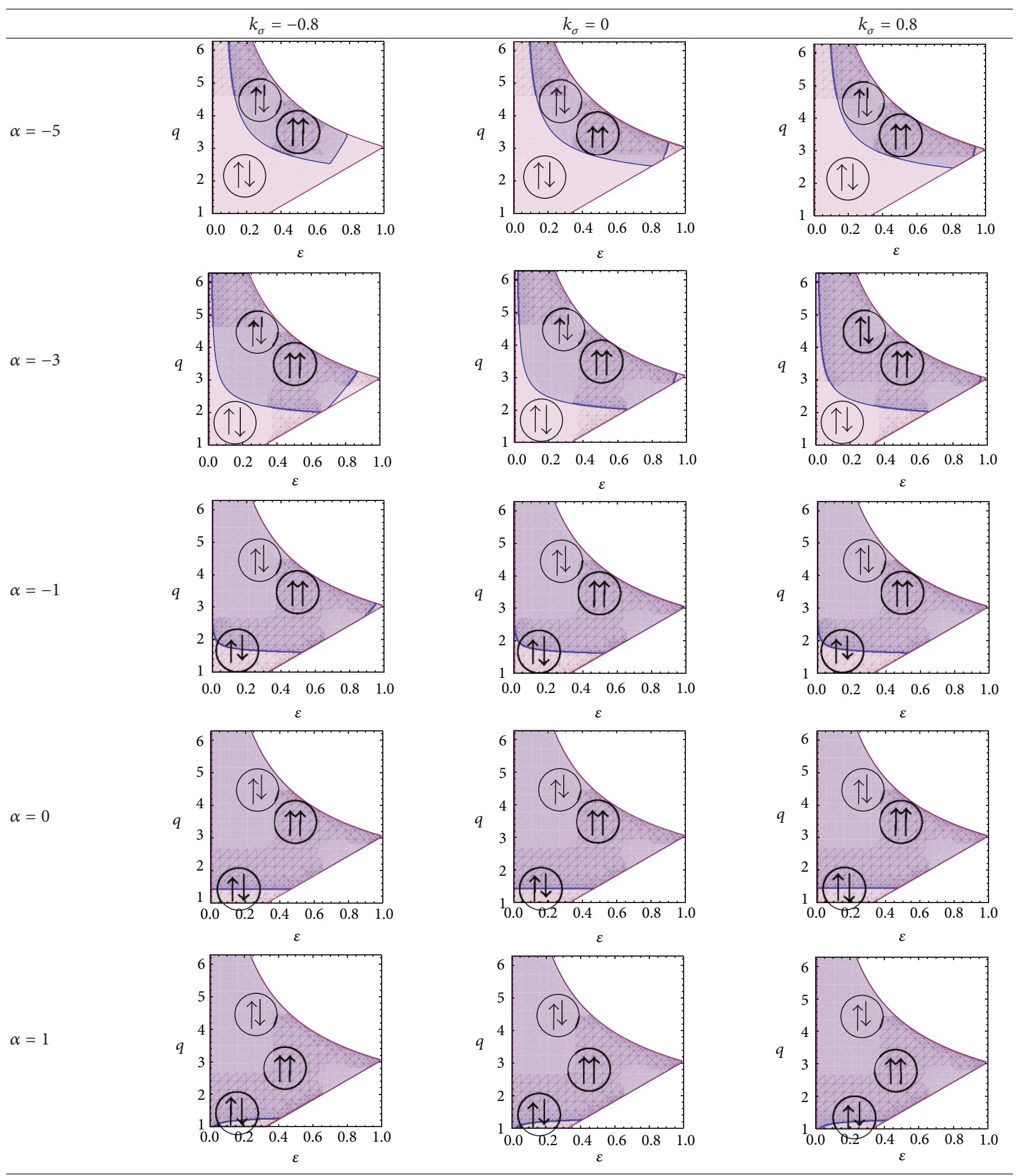


TABLE 2: Continued.

$\alpha=3$

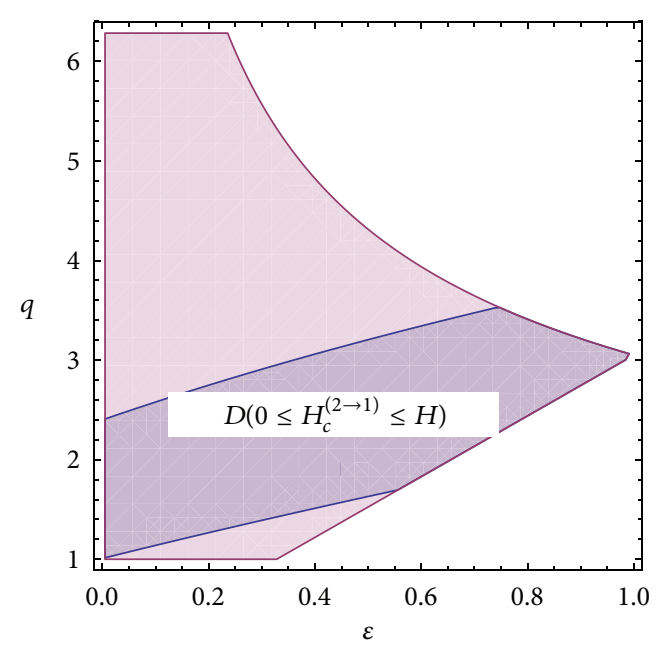

FIGURE 4: Illustration of the calculation of the population of states. The dark region of the picture is area where nanoparticle may move from the first state to the second if the critical field is smaller than the magnetic field.

of nanoparticles $\left(\mathrm{Fe}_{3} \mathrm{O}_{4}-\mathrm{Fe}_{2.44} \mathrm{Ti}_{0.56} \mathrm{O}_{4}\right)$ metastability is more significant (see Table 3 and Figure 3(b)). Tension increases both the degree of metastability and the range of the constants of interfacial exchange interaction $\left[A_{\text {in min }}, A_{\text {in max }}\right]$ where metastable states are implemented (see Figure 3(b)); compression leads to the opposite effect. Described dependence of metastability on mechanical stresses was expected. Since in accordance with the relations (19), an increase in tensile stress increases the effective constant of anisotropy and, therefore, to expansion of the area of metastable states, while compressive stress reduces metastability (see Figure 3(b)).

\section{Magnetization of the System of Two-Phase Nanoparticles}

As shown in the previous section, a two-phase particle in one of the four magnetic states can be represented by a point on a diagram $\{\varepsilon, q\}$ (see Figure 2). We assume that two-phase nanoparticles are uniformly distributed with respect to the relative volume of the second phase $\varepsilon(0 \leq \varepsilon \leq 1)$ and its elongation $q(0 \leq q \leq 2 \pi)$. Then the points representing these nanoparticles are distributed on the diagram $\{\varepsilon, q\}$ uniformly. Therefore, to calculate the dependence of a number of particles $N_{k}(h)(k=1, \ldots 4)$ located in each of the four magnetic states of the magnetic field $h$ we should integrate it with respect to the diagram area $D\left(0 \leq H_{c}^{(i \rightarrow k)} \leq h\right)$ that satisfies conditions $0 \leq H_{c}^{(i \rightarrow k)} \leq h$ (see Figure 4).

So in the case of noninteracting nanoparticles the magnetic states vector population of the two-phase particles $\mathbf{N}(h)=\left\{N_{1}(h), N_{2}(h), N_{3}(h), N_{4}(h)\right\}$ is defined as

$$
\begin{aligned}
N_{1}(h)= & N_{1}(0)+\iint n_{2} D\left(0 \leq H_{c}^{(2 \rightarrow 1)} \leq h\right) d \varepsilon d q \\
& +\iint n_{3} D\left(0 \leq H_{c}^{(3 \rightarrow 1)} \leq h\right) d \varepsilon d q \\
& +\iint n_{4} D\left(0 \leq H_{c}^{(4 \rightarrow 1)} \leq h\right) d \varepsilon d q, \\
N_{2}(h)= & N_{2}(0)+\iint n_{3} D\left(0 \leq H_{c}^{(3 \rightarrow 2)} \leq h\right) d \varepsilon d q \\
& +\iint\left(n_{4}-n_{2}\right) D\left(0 \leq H_{c}^{(2 \rightarrow 4)} \leq h\right) d \varepsilon d q \\
& -\iint n_{2} D\left(0 \leq H_{c}^{(2 \rightarrow 1)} \leq h\right) d \varepsilon d q,
\end{aligned}
$$




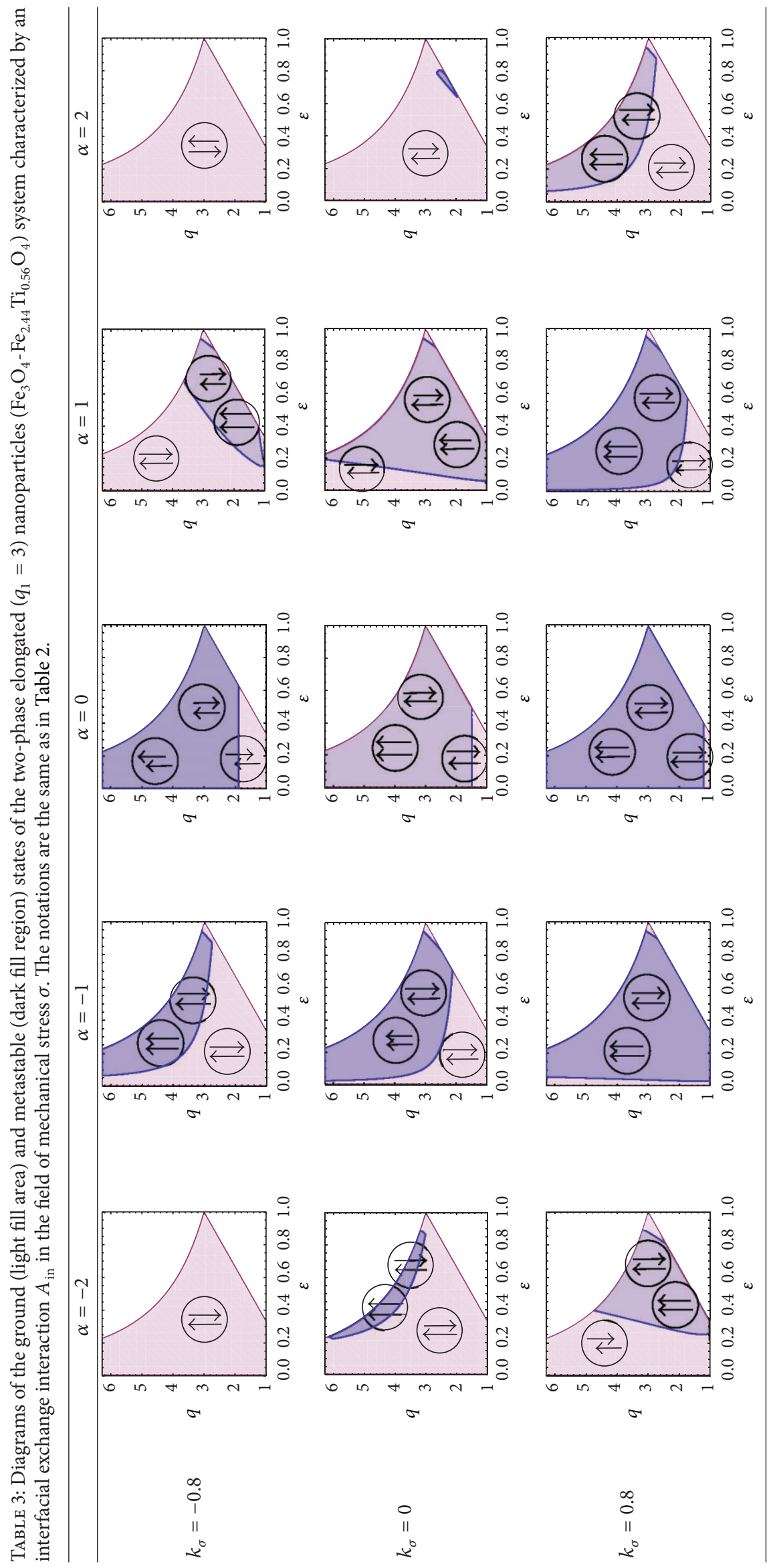




$$
\begin{aligned}
N_{3}(h)= & N_{3}(0)-\iint n_{3} D\left(0 \leq H_{c}^{(3 \rightarrow 1)} \leq h\right) d \varepsilon d q \\
& -\iint n_{3} D\left(0 \leq H_{c}^{(3 \rightarrow 2)} \leq h\right) d \varepsilon d q \\
& -\iint n_{3} D\left(0 \leq H_{c}^{(3 \rightarrow 4)} \leq h\right) d \varepsilon d q, \\
N_{4}(h)= & N_{4}(0)+\iint n_{3} D\left(0 \leq H_{c}^{(3 \rightarrow 4)} \leq h\right) d \varepsilon d q \\
& -\iint n_{4} D\left(0 \leq H_{c}^{(4 \rightarrow 1)} \leq h\right) d \varepsilon d q \\
& -\iint\left(n_{4}-n_{2}\right) D\left(0<H_{c}^{(2 \rightarrow 4)} \leq h\right) d \varepsilon d q .
\end{aligned}
$$

$n_{i}=\left\{n_{1}, \ldots, n_{4}\right\}$ here is the density of distribution on the diagram $\{\varepsilon, q\}$ of a number of particles in $i-M$ state and $N_{i}(0)$ is the number of particles in one of the four equilibrium states at $h=0 . N_{i}(0)$ and $n_{i}$, and they are related as follows:

$$
\begin{aligned}
& N_{1}(0)=\iint n_{1} D\left(0 \leq H_{c}^{(1 \rightarrow 2)}, 0 \leq H_{c}^{(1 \rightarrow 3)}\right. \text {, } \\
& \left.0 \leq H_{c}^{(1 \rightarrow 4)}\right) d \varepsilon d q \\
& +\iint n_{2} D\left(H_{c}^{(2 \rightarrow 1)} \leq 0\right) d \varepsilon d q \\
& +\iint n_{3} D\left(H_{c}^{(3 \rightarrow 1)} \leq 0\right) d \varepsilon d q \\
& +\iint n_{4} D\left(H_{c}^{(4 \rightarrow 1)} \leq 0\right) d \varepsilon d q \\
& N_{2}(0)=\iint n_{1} D\left(H_{c}^{(1 \rightarrow 2)} \leq 0\right) d \varepsilon d q \\
& +\iint n_{2} D\left(0 \leq H_{c}^{(2 \rightarrow 1)}, 0 \leq H_{c}^{(2 \rightarrow 3)},\right. \\
& \left.0 \leq H_{c}^{(2 \rightarrow 4)}\right) d \varepsilon d q \\
& +\iint n_{3} D\left(H_{c}^{(2 \rightarrow 3)} \leq 0\right) d \varepsilon d q \\
& +\iint n_{4} D\left(H_{c}^{(2 \rightarrow 4)} \leq 0\right) d \varepsilon d q \\
& N_{3}(0)=\iint n_{1} D\left(H_{c}^{(1 \rightarrow 3)} \leq 0\right) d \varepsilon d q \\
& +\iint n_{2} D\left(H_{c}^{(2 \rightarrow 3)} \leq 0\right) d \varepsilon d q \\
& +\iint n_{3} D\left(0 \leq H_{c}^{(3 \rightarrow 1)}, 0 \leq H_{c}^{(3 \rightarrow 2)},\right. \\
& \left.0 \leq H_{c}^{(3 \rightarrow 4)}\right) d \varepsilon d q
\end{aligned}
$$

$$
\begin{gathered}
+\iint n_{4} D\left(H_{c}^{(4 \rightarrow 3)} \leq 0\right) d \varepsilon d q \\
N_{4}(0)=\iint n_{1} D\left(H_{c}^{(1 \rightarrow 4)} \leq 0\right) d \varepsilon d q \\
+\iint n_{2} D\left(H_{c}^{(2 \rightarrow 4)} \leq 0\right) d \varepsilon d q \\
+\iint n_{3} D\left(H_{c}^{(3 \rightarrow 4)} \leq 0\right) d \varepsilon d q \\
+\iint n_{4} D\left(0 \leq H_{c}^{(4 \rightarrow 1)}, 0 \leq H_{c}^{(4 \rightarrow 2)}\right. \\
\left.0 \leq H_{c}^{(4 \rightarrow 3)}\right) d \varepsilon d q .
\end{gathered}
$$

$n_{i}$ can be defined from the condition that at $h=0$ in the state of zero magnetization the first and third as well as second and fourth states of nanoparticles are equivalent:

$$
\begin{gathered}
N_{1}(0)=N_{3}(0), \quad N_{2}(0)=N_{4}(0), \\
N_{1}(0)+N_{2}(0)=\frac{N}{2}, \\
n_{1}+n_{2}+n_{3}+n_{4}=\frac{N}{\iint D(\{\varepsilon, q\}) d \varepsilon d q},
\end{gathered}
$$

where $N$ is the number of particles and $\iint D(\{\varepsilon, q\}) d \varepsilon d q$ is the total area of the diagram $\{\varepsilon, q\}$ (see Figure 2).

Relations (21)-(23) defining the population vector $\mathbf{N}(h)$ allow for calculating the value of magnetization of the twophase nanoparticles system:

$$
\begin{aligned}
I(h)=c[ & \left(N_{1}(h)-N_{3}(h)\right)\left((1-\varepsilon) I_{s}^{(1)}+\varepsilon I_{s}^{(2)}\right) \\
& \left.+\left(N_{2}(h)-N_{4}(h)\right)\left((1-\varepsilon) I_{s}^{(1)}-\varepsilon I_{s}^{(2)}\right)\right],
\end{aligned}
$$

where $c=N V / V_{0}$ is the volume concentration of the magnetic phase and $V_{0}$ is the volume that the system occupies.

\section{The Effect of Mechanical Stress on the Magnetization Curve and Hysteresis Characteristics of an Ensemble of Cobalt-Coated Nanoparticles $\gamma-\mathrm{Fe}_{2} \mathrm{O}_{3}$}

Calculation of the magnetization curve and hysteresis loop held with the help of relations (11)-(16) and (21)-(24) is shown in Figure 5.

Dependence of the coercive force $H_{c}$ on the relative volume of cobalt coating $\tau=1-\varepsilon$ is determined not only by mechanical stress but also by the interfacial exchange $A_{\text {in }}$ (see Figure 6).

It is easy to see that tension shifts magnetization curves to lower magnetic $H$, but compression has the opposite effectmagnetization curves shift to larger fields $H$ relatively to the undeformed state. Thus, the mechanical stress does not affect the saturation magnetization $I_{s}$, which coincides with the 

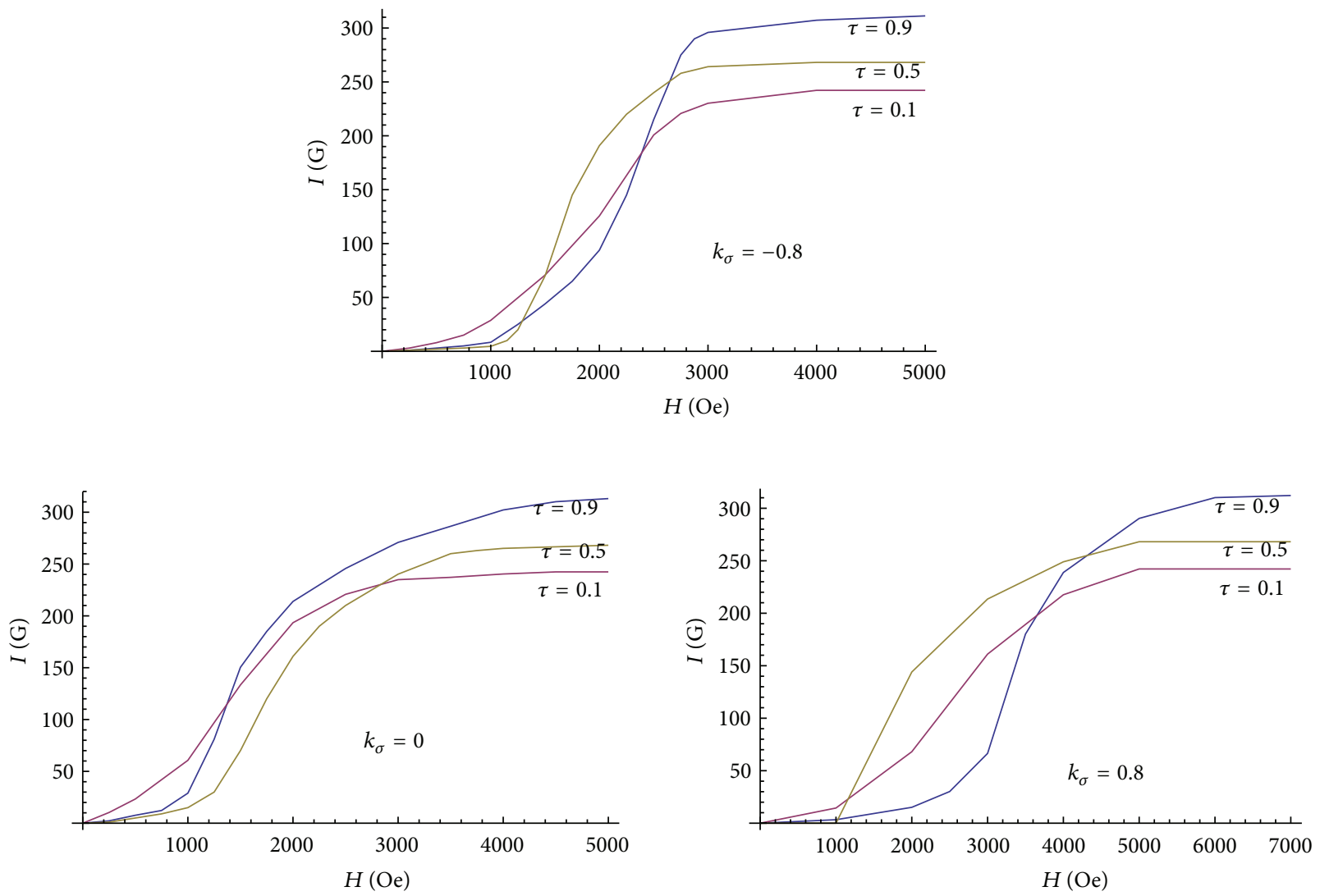

Figure 5: Dependence of magnetization of the elongated nanoparticles system (with the following characteristics: $q_{1}=3, A_{\text {in }}=0$ ) on the magnetic field $H$, relative volume of the cobalt coating $\tau=1-\varepsilon$, and relative mechanical stress $k_{\sigma}$.

saturation remanence $I_{\mathrm{rs}}$ and decreases with increasing thickness of the membrane irritation. Recent experiments conducted on the core/shell nanoparticles of hematite/magnetite showed that $I_{s}$ is almost independent of stress [68]. We obtained that the dependence of the saturation magnetization of the relative amount of cobalt coating $\tau$ is in qualitative agreement with experimental data [69]. $I_{s}$ reduction system core/shell nanoparticles with increasing thickness of the shell due to the fact that with increasing $\tau$ is increased contribution less magnetic material and a magnetic role decline.

Dependence of the coercive force $H_{c}$ on the relative volume of cobalt coating $\tau=1-\varepsilon$ is determined not only by mechanical stress but also by the interfacial exchange $A_{\text {in }}$ (see Figure 6). In addition, the negative exchange interaction leads to a reduction in general, and the positive increases $H_{c}$ relative to the state $A_{\text {in }}=0$. We calculated the dependence of the coercive force of $\tau$ which is in qualitative agreement with experimental data [70]. Where shown, the dependence of the coercive force is not determined by the particle size and the relative thickness of coating core/shell nanoparticles.

We also note a qualitative agreement with experimental data [68]; we obtained depending $H_{c}$ from mechanical stresses. In the same paper was received experimental linear dependence of the effective anisotropy constant of stress which is in good agreement with the results obtained by us (see (19)). Dependence characteristics of the coercive force on the interfacial exchange interaction $A_{\text {in }}$ are shown in Figure 7. Obviously, the nonmonotonic behavior $H_{c}=$ $H_{c}\left(A_{\text {in }}\right)$ is typical for nanoparticles with a large $(\tau=0.9)$ or low $(\tau=0.1)$ cobalt coating thickness and implemented in both positive and negative values $A_{\text {in }}$.

Since the coercive force is determined by the minimum of the range of critical fields, the nonmonotonic behavior $H_{c}$ is related to the difference in the dependence of these fields on the exchange interaction constant $A_{\text {in }}$ (see (10) and (11)-(16)). The above mentioned nonmonotonic behavior $H_{c}$ was not observed in $[39,65,66,71]$, which is obviously associated with a narrower range of critical fields of magnetization reversal of two-phase particles than that in this study. A qualitative comparison of the results with the similar calculations presented in the works $[8,54,69,71,72]$ shows that coercivity increases up to saturation along with the increase in the amount of $\mathrm{CoFe}_{2} \mathrm{O}_{4}$ phase, just as it was mentioned in those works.

\section{Conclusions}

In contrast to earlier models of two-phase nanoparticles [56$59,67,73]$ the model presented in this work allows for studying the effect of mechanical stress and magnetic field on the magnetic state of both uniaxis and multiaxis heterophase 

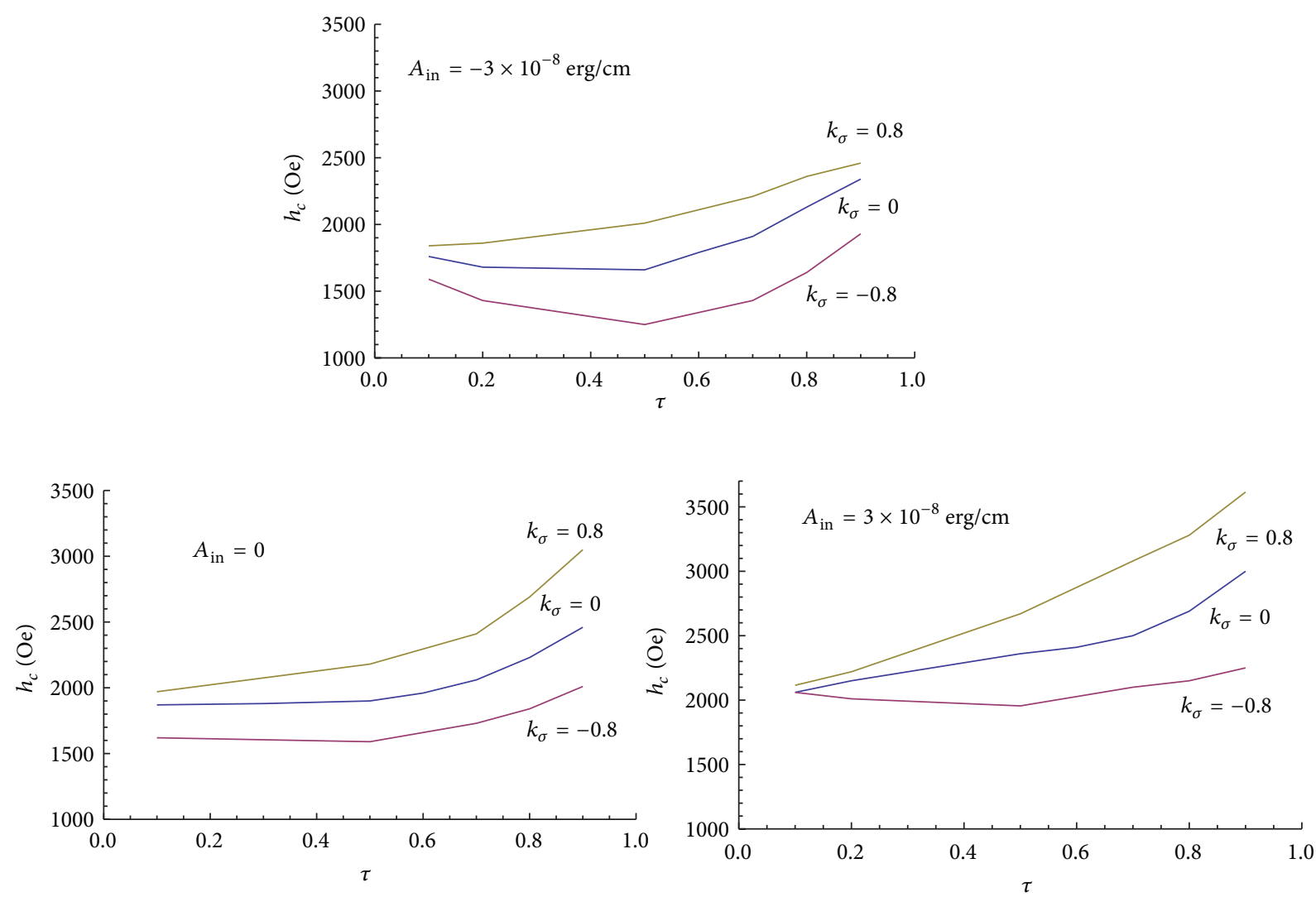

FiguRE 6: Dependence of the relative coercive force $h_{c}=H_{c}(\sigma) / H_{c m}(\sigma=0)$ of the elongated nanoparticles system $\left(q_{1}=3, H_{c m}(\sigma=0)=\right.$ 2947 Oe) on the relative amount of cobalt coating $\tau$, relative mechanical stress $k_{\sigma}$, and the interfacial exchange $A_{\text {in }}$.
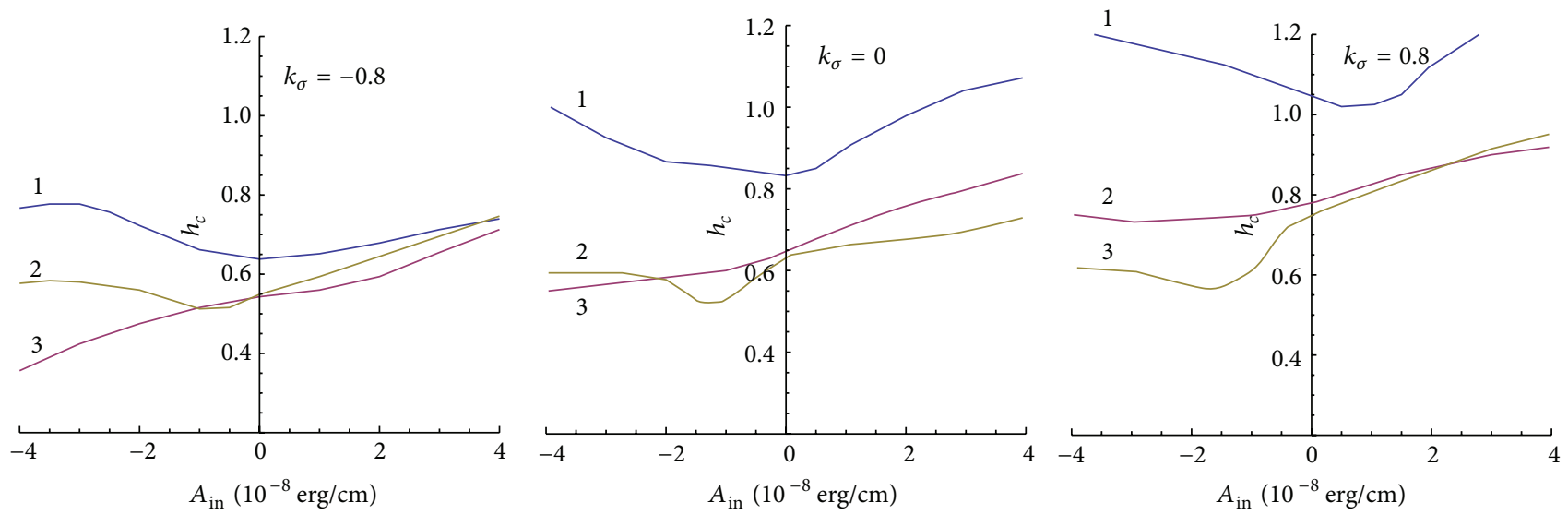

FIGURE 7: Dependence of the relative coercive force $h_{c}=H_{c} / H_{c 1}$ of the elongated nanoparticles system (with the following characteristics: $q_{1}=3, H_{c m}=2947 \mathrm{Oe}$ ) on the value of the interfacial exchange $A_{\text {in }}$, relative volume of cobalt coating $\tau$, and relative mechanical stress, 1 $-\tau=0.9,2-\tau=0.1,3-\tau=0.5$.

magnets. The estimated range of the critical fields of magnetization reversal of the phases' magnetic moments allowed for building phase diagrams and evaluating the effect of mechanical stress on the degree of metastability of nanoparticles' magnetic states. In the framework of our model, using an example of epitaxially cobalt-coated particles $\gamma-\mathrm{Fe}_{2} \mathrm{O}_{3}$ we carried out a theoretical analysis of the effect of uniaxial stress on the magnetization process of the system of noninteracting heterophase nanoparticles. As it was shown tension leads to reduction and compression increases coercive force $H_{c}$, while the residual saturation magnetization $I_{\text {rs }}$ does not change under the influence of mechanical stress. The coercive force 
of the nanoparticles system changes in a nonmonotonic way as far as the interfacial exchange interaction increases and strongly depends on the thickness of cobalt coating.

The simulation of the effect of mechanical stresses on the magnetization of the system of two-phase singledomain noninteracting grains carried out in this study led to consistent results, which allows for using our model in analyzing magnetic properties of an ensemble of heterophase nanoparticles.

\section{References}

[1] F. D. Stacey and S. K. Banerjee, The Physical Principles of Rock Magnetism, Elsevier, New York, NY, USA, 1974.

[2] V. I. Bagin, T. S. Gendler, and T. A. Avilova, The Magnetism of Iron Oxides and Hydroxides, Publishing House of the USSR Academy of IPE, Moscow, Russia, 1988.

[3] A. K. Gapeev and V. A. Tselmovich, "Composition heterogeneously oxidized natural and synthetic titanomagnetite," Physics of the Earth, no. 10, pp. 42-48, 1988.

[4] A. K. Gapeev and V. A. Tselmovich, "The microstructure of natural heterogeneously oxidized titanomagnetite," Izvestia ASUSSR, Physics of the Earth, no. 4, pp. 100-101, 1986.

[5] T. G. Artemov and A. K. Gapeev, "On the decomposition of solid solutions in the system magnetite-ulvospinel," Izvestia ASUSSR, Physics of the Earth, no. 12, pp. 82-87, 1988.

[6] X. Wei, R. Skomski, B. Balamurugan, and D. J. Sellmyer, "Magnetism of core-shell $\mathrm{Ti}$ : TiO nanoparticles," Journal of Applied Physics, vol. 107, no. 9, Article ID 09B516, 3 pages, 2010.

[7] L. Wang, H. Park, S. I. Lim et al., "Core@shell nanomaterials: gold-coated magnetic oxide nanoparticles," Journal of Materials Chemistry, vol. 18, no. 23, pp. 2629-2635, 2008.

[8] A. E. Kandjani, S. E. H. Amiri, M. R. Vaezi, and S. K. Sadrnezhaad, "Optical and magnetic properties of $\mathrm{Co}_{3} \mathrm{O}_{4} / \mathrm{ZnO}$ core/shell nanoparticles," Journal of Optoelectronics and Advanced Materials, vol. 12, no. 10, pp. 2057-2062, 2010.

[9] T. Wen and K. M. Krishnan, "Magnetic properties of $\mathrm{Au}_{\text {core }}{ }^{-}$ $\mathrm{Co}_{\text {shell }}$ nanoparticles," Journal of Applied Physics, vol. 109, no. 7, Article ID 07B515, 3 pages, 2011.

[10] Y. S. Koo, D. H. Kim, and J. H. Jung, "Synthesis of electric/magnetic oxide core/shell nanoparticles and their characteristics," Journal of the Korean Physical Society, vol. 48, no. 4, pp. 677-680, 2006.

[11] L. Y. Lu, D. Wang, X. G. Xu, H. C. Wang, J. Miao, and Y. Jiang, "Low temperature magnetic hardening in self-assembled FePt/Ag core-shell nanoparticles," Materials Chemistry and Physics, vol. 129, no. 3, pp. 995-999, 2011.

[12] S. Chandra, H. Khurshid, M. H. Phan, and H. Srikanth, "Asymmetric hysteresis loops and its dependence on magnetic anisotropy in exchange biased $\mathrm{Co} / \mathrm{CoO}$ core-shell nanoparticles," Applied Physics Letters, vol. 101, no. 23, Article ID 232405, 5 pages, 2012.

[13] H. Khurshid, S. Chandra, W. Li et al., "Synthesis and magnetic properties of core/shell $\mathrm{FeO} / \mathrm{Fe}_{3} \mathrm{O}_{4}$ nano-octopods," Journal of Applied Physics, vol. 113, no. 17, Article ID 17B508, 3 pages, 2013.

[14] M. Gao, W. Li, J. Dong, Z. Zhang, and B. Yang, "Synthesis and characterization of superparamagnetic $\mathrm{Fe}_{3} \mathrm{O}_{4} @ \mathrm{SiO}_{2}$ core-shell composite nanoparticles," World Journal of Condensed Matter Physics, vol. 1, no. 2, pp. 49-54, 2011.

[15] J. H. Li, R. Y. Hong, H. Z. Li, J. Ding, Y. Zheng, and D. G. We, "Simple synthesis and magnetic properties of $\mathrm{Fe}_{3} \mathrm{O}_{4} / \mathrm{BaSO}_{4}$ multi-core/shell particles," Materials Chemistry and Physics, vol. 113, no. 1, pp. 140-144, 2009.

[16] W. Fang, X. Chen, and N. Zheng, "Superparamagnetic coreshell polymer particles for efficient purification of his-tagged proteins," Journal of Materials Chemistry, vol. 20, no. 39, pp. 8624-8630, 2010.

[17] K. S. Kumar, V. B. Kumar, and P. Paik, "Recent advancement in functional core-shell nanoparticles of polymers: synthesis, physical properties, and applications in medical biotechnology," Journal of Nanoparticles, vol. 2013, Article ID 672059, 24 pages, 2013.

[18] Z. Sen, J. Lee, and S. Sun, "Controlled synthesis of monodisperse magnetic nanoparticles in solution phase," The Open Surface Science Journal, vol. 4, pp. 26-34, 2012.

[19] J. H. Li, R. Y. Hong, H. Z. Li, J. Ding, Y. Zheng, and D. G. Wei, "Simple synthesis and magnetic properties of $\mathrm{Fe}_{3} \mathrm{O}_{4} / \mathrm{BaSO}_{4}$ multi-core/shell particles," Materials Chemistry and Physics, vol. 113, no. 1, pp. 140-144, 2009.

[20] S. Bethrens, H. Bonnermann, N. Matoussevitch et al., "Airstable Co-, Fe-, and Fe/Co-nanoparticles and ferrofluids," Zeitschrift für Physikalische Chemie, vol. 220, no. 1, pp. 3-40, 2006.

[21] H. Y. Park, M. J. Schadt, L. Wang et al., "Fabrication of magnetic core@Shell Fe oxide@ Au nanoparticles for interfacial bioactivity and bio-separation," Langmuir, vol. 23, no. 17, pp. 9050-9056, 2007.

[22] W. Wu, Q. He, and C. Jiang, "Magnetic iron oxide nanoparticles: synthesis and surface functionalization strategies," Nanoscale Research Letters, vol. 3, no. 11, pp. 397-415, 2008.

[23] F. Frederix, J. M. Friedt, K. H. Choi et al., "Biosensing based on light absorption of nanoscaled gold and silver particles," Analytical Chemistry, vol. 75, no. 24, pp. 6894-6900, 2003.

[24] S. Praharaj, S. Nath, S. K. Ghosh, S. Kundu, and T. Pal, "Immobilization and recovery of Au nanoparticles from anion exchange resin: resin-bound nanoparticle matrix as a catalyst for the reduction of 4-nitrophenol," Langmuir, vol. 20, no. 23, pp. 9889-9892, 2004.

[25] C. T. Campbell, S. C. Parker, and D. E. Starr, "The effect of size-dependent nanoparticle energetics on catalyst sintering," Science, vol. 298, no. 5594, pp. 811-814, 2002.

[26] M. Mahmoudi and K. S. Suslick, "Protein fibrillation and the olfactory system: speculations on their linkage," Trends in Biotechnology, vol. 30, no. 12, pp. 609-610, 2012.

[27] J. Chomoucka, J. Drbohlavova, D. Huska, V. Adam, R. Kizek, and J. Hubalek, "Magnetic nanoparticles and targeted drug delivering," Pharmacological Research, vol. 62, no. 2, pp. 144149, 2010.

[28] N. K. Verma, K. Crosbie-Staunton, A. Satti et al., "Magnetic core-shell nanoparticles for drug delivery by nebulization," Journal of Nanobiotechnology, vol. 11, article 1, 2013.

[29] J. Neamtu and N. Verga, "Magnetic nanoparticles for magnetoresonance imaging and targeted drug delivery," Digest Journal of Nanomaterials and Biostructures, vol. 6, no. 3, pp. 969-978, 2011.

[30] S. Prijic and G. Sersa, "Magnetic nanoparticles as targeted delivery systems in oncology," Radiology and Oncology, vol. 45, no. 1, pp. 1-16, 2011.

[31] S. Purushotham, P. E. J. Chang, H. Rumpel et al., "Thermoresponsive core-shell magnetic nanoparticles for combined modalities of cancer therapy," Nanotechnology, vol. 20, no. 30, Article ID 305101, 2009. 
[32] T. Y. Liu, S. H. Hu, D. Liu, S. Chen, and I. Chen, "Biomedical nanoparticle carriers with combined thermal and magnetic responses," Nano Today, vol. 4, no. 1, pp. 52-65, 2009.

[33] T. A. Skotheim and M. G. Kanatzidis, "Polymeric electrical conductors," Chemical and Engineering News, vol. 1990, pp. 36$54,1990$.

[34] P. Andersson, R. Forchheimer, P. Tehrani, and M. Berggren, "Printable all-organic electrochromic active-matrix displays," Advanced Functional Materials, vol. 17, no. 16, pp. 3074-3082, 2007.

[35] Y. Liu and T. Cui, "Polymeric integrated AC follower circuit with a JFET and an ink-jet printed resistor as active devices," Solid State Electronics, vol. 49, no. 3, pp. 445-448, 2005.

[36] K. Haneda and A. H. Morrish, "Mössbauer study of Fe-oxide surface layers formed on small Fe particles," Surface Science, vol. 77, no. 3, pp. 584-590, 1978.

[37] V. Papaefthymiou, A. Kostikas, A. Simopoulos et al., "Magnetic hysteresis and Mössbauer studies in ultrafine iron particles," Journal of Applied Physics, vol. 67, no. 9, pp. 4487-4489, 1990.

[38] S. J. Cho, S. M. Kauzlarich, J. Olamit et al., "Characterization and magnetic properties of core/shell structured Fe/Au nanoparticles," Journal of Applied Physics, vol. 95, no. 11, pp. 6804-6806, 2004.

[39] J. S. Yang and C. R. Chang, "Magnetization curling in elongated heterostructure particles," Physical Review B, vol. 49, no. 17, pp. 11877-11885, 1994.

[40] M. Grigorova, H. J. Blythe, V. Blaskov et al., "Magnetic properties and Mössbauer spectra of nanosized $\mathrm{CoFe}_{2} \mathrm{O}_{4}$ powders," Journal of Magnetism and Magnetic Materials, vol. 183, no. 1-2, pp. 163-172, 1998.

[41] M. Kishimoto, S. Kitaoko, H. Andoh, M. Amemiya, and F. Hayama, "On the coercivity of cobalt-ferrite epitaxial iron oxides," IEEE Transactions on Magnetics, vol. 17, no. 6, pp. 30293031, 1981.

[42] G. Salazar-Avarez, J. Qin, V. Sepelak et al., "Cubic versus spherical magnetic nanoparticles: the role of surface anisotropy," Journal of the American Chemical Society, vol. 130, no. 40, pp. 13234-13239, 2008.

[43] Q. Song and Z. J.Zhang, "Shape control and associated magnetic properties of spinel cobalt ferrite nanocrystals," Journal of the American Chemical Society, vol. 126, no. 19, pp. 6164-6168, 2004.

[44] M. E. Schabes and H. N. Rertram, "Magnetization reversal of cobalt-modified $\gamma$ - $\mathrm{Fe}_{2} \mathrm{O}_{3}$ particles," Journal of Applied Physics, vol. 67, no. 11, article 5149, 3 pages, 1990.

[45] A. E. Berkowitz, F. E. Parker, E. L. Hall, and G. Podolsky, "Toward a model for Co-surface-treated Fe-oxides," IEEE Transactions on Magnetics, vol. 24, no. 6, pp. 2871-2873, 1988.

[46] T. Iglesias, A. Labarta, and X. Batlle, "Exchange bias phenomenology and models of core/shell nanoparticles," Journal of Nanoscience and Nanotechnology, vol. 8, no. 6, pp. 2761-2780, 2008.

[47] K. Khandrikh and C. Kobe, Amorphous Ferro- and Ferrimagnets: Translation from Germ, Mir Publishers, Moscow, Russia, 1982.

[48] I. V. Zolotukhin and Y. E. Kalinin, "Amorphous metallic alloys," Advances in Physical Sciences, vol. 33, no. 9, pp. 720-738, 1990.

[49] Y. I. Ustinovshchikov and B. E. Pushkarev, "Ordering, phase separation, and phase transformations in Fe-M alloys," PhysicsUspekhi, vol. 49, no. 6, pp. 593-603, 2006.

[50] A. A. Zisman and A. A. Vasiliev, "The phase voltages induced $\gamma \rightarrow \alpha$ transformation in a polycrystalline iron," Solid State Physics, vol. 46, no. 11, pp. 2051-2055, 2004.
[51] Y. Komorida, M. Mito, H. Deguchi et al., "Effect of large mechanical stress on the magnetic properties of embedded Fe nanoparticles," Beilstein Journal of Nanotechnology, vol. 2, pp. 268-275, 2011.

[52] H. Jeen and A. Biswas, "Single domain to multidomain transition due to in-plane magnetic anisotropy in phase-separated $\left(\mathrm{La}_{0.4} \mathrm{Pr}_{0.6}\right)_{0.67} \mathrm{Ca}_{0.33} \mathrm{MnO}_{3}$ thin films," Physical Review B, vol. 83, no. 6, Article ID 064408, 8 pages, 2011.

[53] J. Zhu, S. Wei, J. Ryu, L. Sun, Z. Luo, and Z. Guo, "Magnetic epoxy resin nanocomposites reinforced with core-shell structured Fe@FeO nanoparticles: fabrication and property analysis," ACS Applied Materials and Interfaces, vol. 2, no. 7, pp. 2100-2107, 2010.

[54] H. Jeen and A. Biswas, "Single domain to multidomain transition due to in-plane magnetic anisotropy in phase-separated $\left(\mathrm{La}_{0.4} \mathrm{Pr}_{0.6}\right)_{0.67} \mathrm{Ca}_{0.33} \mathrm{MnO}_{3}$ thin films," Physical Review B, vol. 83, no. 6, Article ID 064408, 8 pages, 2011.

[55] Y. Komorida, M. Mito, H. Deguchi et al., "Surface and core magnetic anisotropy in maghemite nanoparticles determined by pressure experiments," Applied Physics Letters, vol. 94, no. 20, Article ID 202503, 3 pages, 2009.

[56] L. L. Afremov and A. V. Panov, Residual Magnetization of Ultrafine Magnets, FESTU Press, Vladivostok, Russia, 2004.

[57] J. S. Yang and C. R. Chang, "The influence of interfacial exchange on the coercivity of acicular coated particle," Journal of Applied Physics, vol. 69, no. 11, article 7756, 6 pages, 1991.

[58] L. L. Afremov and A. V. Panov, "Theory of magnetization of two-phase superparamagnetic particles: I. Magnetic states," Physics of Metals and Metallography, vol. 82, no. 5, pp. 439-444, 1996.

[59] L. L. Afremov and A. V. Panov, Physics of Metals and Metallography, vol. 82, no. 5, pp. 445-448, 1996.

[60] Y. Syono, Japanese Journal of Geophysics, vol. 4, no. 1, pp. 71-143, 1965.

[61] S. Krupichka, Physics of Ferrites and Related Magnetic Oxides, vol. 2 of edited by A. Pakhomov, 1976.

[62] S. Tikadzumi, Physics of Ferromagnetism: Magnetic Characteristics and Practical Applications, Mir Publishers, Moscow, Russia, 1987.

[63] Y. Ghen, J. E. Snyder, C. R. Schwichtenberg, and K. W. Dennis, "Metal-bonded Co-ferrite composites for magnetostrictive torque sensor applications," IEEE Transactions on Magnetics, vol. 35, no. 5, pp. 3652-3654, 1999.

[64] D. L. Hou, X. F. Nie, and H. L. Luo, "Studies on the magnetic viscosity and the magnetic anisotropy of $\gamma-\mathrm{Fe}_{2} \mathrm{O}_{3}$ powders," Applied Physics A, vol. 66, no. 1, pp. 109-114, 1998.

[65] J. Smith and H. Wayne, Ferrites, Foreign Literature Publishing House, Moscow, Russia, 1962.

[66] M. J. Stavn and A. H. Morrish, "Magnetization of a twocomponent Stoner-Wohlfarth particle," IEEE Transactions on Magnetics, vol. 15, no. 5, pp. 1235-1240, 1979.

[67] L. L. Afremov and A. V. Panov, "Effect of mechanical stresses on the saturation remanence of a system of nanoparticles," Physics of Metals and Metallography, vol. 106, no. 3, pp. 238-246, 2008.

[68] N. J. O. Silva, S. Saisho, M. Mito et al., "Pressure effects in hollow and solid iron oxide nanoparticles," http://arxiv.org/abs/ 1301.5708.

[69] G. Salazar-Alvarez, J. Sort, S. Suriñach, M. D. Baró, and J. Nogués, "Synthesis and size-dependent exchange bias in inverted core-shell $\mathrm{MnO}-\mathrm{Mn}_{3} \mathrm{O}_{4}$ nanoparticles," Journal of the American Chemical Society, vol. 129, no. 29, pp. 9102-9108, 2007. 
[70] H. Zeng, S. Sun, J. Li, Z. L. Wang, and J. P. Liu, "Tailoring magnetic properties of core/shell nanoparticles," Applied Physics Letters, vol. 85, no. 5, pp. 792-794, 2004.

[71] O. Iglesias, A. Labarta, and X. Batlle, "Exchange bias phenomenology and models of core/shell nanoparticles," Journal of Nanoscience and Nanotechnology, vol. 8, no. 6, pp. 2761-2780, 2008.

[72] A. Aharoni, "Magnetization buckling in elongated particles of coated iron oxides," Journal of Applied Physics, vol. 63, no. 9, pp. 4605-4616, 1988.

[73] L. L. Afremov and A. V. Panov, "Simulating the magnetization of an ensemble of cobalt-coated $\gamma-\mathrm{Fe}_{2} \mathrm{O}_{3}$ particles," Physics of Metals and Metallography, vol. 87, no. 1, pp. 12-16, 1999. 

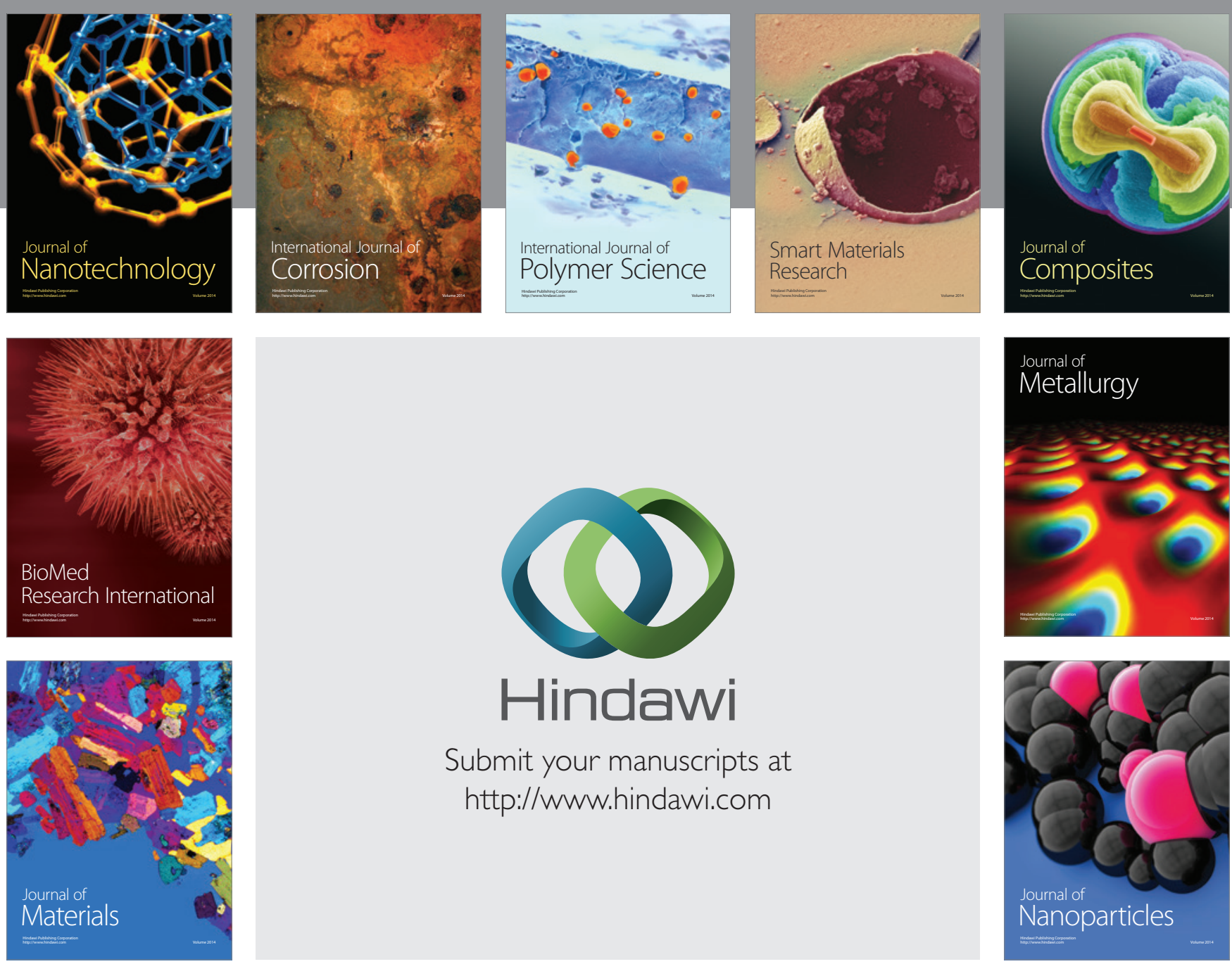

Submit your manuscripts at http://www.hindawi.com
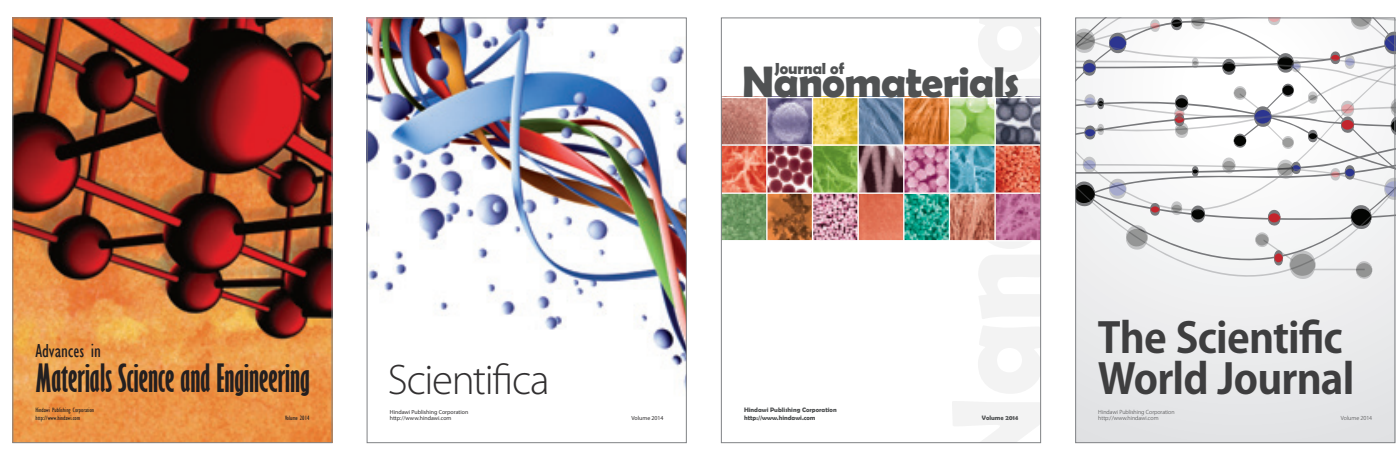

\section{The Scientific World Journal}
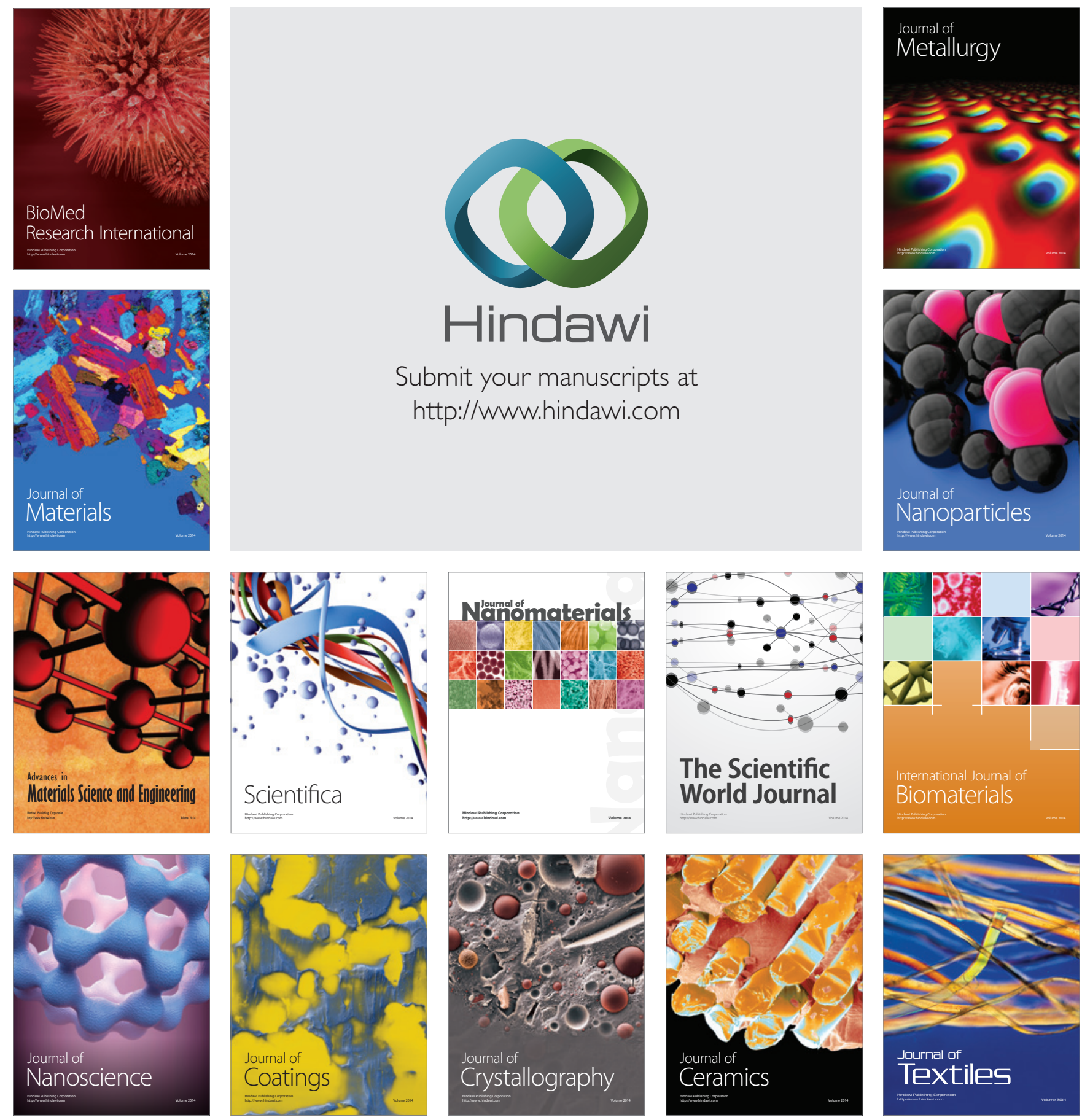